\title{
Fauna edáfica e suas relações com atributos químicos, físicos e microbiológicos em Floresta de Araucária
}

\author{
Edaphic fauna and its relations with chemical, physical and microbiological \\ attributes in Araucaria forest
}

\begin{abstract}
Jamil de Morais Pereira', Dilmar Baretta" ${ }^{\mathrm{II}}$ Luís Carlos Iuñes de Oliveira Filho"II, Carolina Riviera Duarte Maluche-Baretta ${ }^{\mathrm{Iv}}$, Elke Jurandy Bran Nogueira Cardoso ${ }^{\mathrm{v}}$
\end{abstract}

\begin{abstract}
Resumo
A permanente cobertura vegetal do solo, em Florestas de Araucária, contribui na conservação de sua biodiversidade edáfica. O objetivo deste trabalho foi avaliar o potencial da fauna edáfica e das variáveis ambientais explicativas (físico-químicas e microbiológicas do solo) na discriminação de florestas com araucária nativa e reflorestada. Avaliaram-se florestas com Araucaria angustifolia nativa (NF) e reflorestada (RF) em três regiões distintas no estado de São Paulo, representando três repetições. Em cada área, 15 amostras de solo foram coletadas para avaliação dos atributos físicos, químicos e microbiológicos e, nos mesmos pontos, procedeu-se às coletas da fauna utilizando-se o método de armadilhas de queda (Pitfall traps). A fauna do solo foi influenciada pela sazonalidade, apresentando maior abundância de indivíduos no verão. Os grupos Collembola, Formicidae e Coleoptera foram os mais abundantes, independentemente da época de amostragem e tipo de floresta. A Análise de Componentes Principais (ACP) e Análise Canônica Discriminante (ACD) claramente diferenciaram as áreas de araucárias. Na ACP, Hemiptera, Collembola e Diplopoda ficaram associados à RF no inverno, principalmente pelos maiores valores de matéria seca da serapilheira. Já em NF, foram os grupos Orthoptera, Hymenoptera, Araneae e Coleoptera, explicados pela melhor qualidade do solo e da serapilheira. No verão, ficaram associados à NF, os grupos Formicidae, Hemiptera, Orthoptera, Araneae, Coleoptera e Collembola, explicados pelos maiores valores de CBM, Ca, P, C-org, macroporosidade e atividade de desidrogenase. Na ACD, a abundância de grupos taxonômicos foi o atributo da fauna edáfica mais importante para a discriminação das florestas. Da mesma forma, a umidade do solo, teor de $\mathrm{P}$ no solo, porosidade total e teor de $\mathrm{S}$ na serapilheira contribuíram na discriminação das florestas. A semelhança entre as áreas, em relação aos grupos da fauna edáfica, indica que está ocorrendo uma estabilidade das áreas reflorestadas comparável às nativas.
\end{abstract}

Palavras-chave: Floresta nativa e reflorestada; Análise multivariada; Invertebrados de solo; Biodiversidade do solo

Engenheiro Agrônomo, Dr., Professor do Instituto Federal de Educação, Ciência e Tecnologia do Sul de Minas Gerais, Praça Tiradentes, 416, CEP 37576-000, Inconfidentes (MG), Brasil. jamilmpereira@gmail.com (ORCID: 0000-0002-3191-2251)

Engenheiro Agrônomo, Dr., Professor Associado do Centro de Educação Superior do Oeste, Universidade do Estado de Santa Catarina, Rua Beloni Trombeta Zanin, 680E, CEP 89815-630, Chapecó (SC), Brasil. dilmar.baretta@udesc.br (ORCID: 0000-0001-8219-1362)

III Engenheiro Agrônomo, Dr., Pós-Doutorando do Centro de Educação Superior do Oeste, Universidade do Estado de Santa Catarina, Rua Beloni Trombeta Zanin, 680E, CEP 89815-630, Chapecó (SC), Brasil. iunes1981@gmail.com (ORCID: 0000-0002-9010-481X)

Engenheira Agrônoma, Drª., Professora Titular da Universidade Comunitária da Região de Chapecó, Servidão Anjo da Guarda, 295-D, CEP 89809900, Chapecó (SC), Brasil. carolmaluche@unochapeco.edu.br (ORCID: 0000-0001-7131-1517)

Engenheira Agrônoma, Drª., Professora Titular Sênior da Escola Superior de Agricultura “Luiz de Queiroz”, Universidade de São Paulo, Av. Pádua Dias, CEP 13418-900, Piracicaba (SP), Brasil. ejbncard@usp.br (ORCID: 0000-0003-3938-0373) 


\begin{abstract}
The permanent plant cover of the soil, in Araucaria forests, contributes to the conservation of the soil biodiversity. The aim of this study was to evaluate the potential of the soil fauna in discriminating native and reforested Araucaria forests. Native (NF) and reforested (RF) Araucaria angustifolia forests were evaluated in three regions of the state of São Paulo, representing three true replicates. In each area, fifteen soil samples were collected for the evaluation of the physical, chemical, and microbiological attributes and at the same points, the fauna was collected, using pitfall traps. The soil fauna was influenced by seasonality, presenting greater abundance of individuals in the summer. Collembola, Formicidae and Coleoptera groups were the most abundant ones, independently of the sampling season and the forest type. The Principal Component Analysis (PCA) and Canonical Discriminant Analysis (CDA) clearly differentiated native or reforested Araucaria areas. In PCA, Hemiptera, Collembola and Diplopoda were associated with RF in the winter, mainly due to the higher litter dry mass values. In NF, Orthoptera, Hymenoptera, Araneae and Coleoptera groups were explained by the higher quality of soil and litter, which was associated with NF. In summer, the groups Hemiptera, Orthoptera, Araneae, Coleoptera and Collembola, associated with NF, were explained by the higher values of microbial biomass carbon, $\mathrm{Ca}, \mathrm{P}$, organic carbon, macroporosity and dehydrogenase activity. In the CDA, the abundance of taxonomic groups was the most important attribute of the soil fauna for the discrimination of the forests. Likewise, soil moisture, soil P content, total porosity, and sulfur content of the surface litter contributed to discrimination between forests. There was some similarity between areas regarding certain groups of soil fauna, which we suggest being a result of the stability reached by the reforested areas, rendering them somewhat similar to the native ones.
\end{abstract}

Keywords: Native and reforested forests; Multivariate analysis; Soil invertebrates; Soil biodiversity

\title{
Introdução
}

A Floresta Ombrófila Mista (Floresta de Araucária), no Brasil, tem como principal representante a Araucaria angustifolia (Bertoloni) Otto Kuntze (Araucariaceae). Sua ocorrência é mais restrita aos estados do Rio Grande do Sul, Santa Catarina e Paraná, embora sejam encontrados segmentos esparsos dessa floresta também nos estados de São Paulo, Minas Gerais e Rio de Janeiro (HUECK, 1972). A Floresta de Araucária já foi muito exuberante, cobrindo cerca de $200.000 \mathrm{~km}^{2}$, embora só restem 2 a 4\% dessa área (GUERRA et al., 2002; RIBEIRO et al., 2009).

No estado de São Paulo, o solo sob Floresta de Araucária concentra alta diversidade de invertebrados edáficos (BARETTA; BROWN; CARDOSO, 2010; PEREIRA; BARETTA; CARDOSO, 2015) dependentes dessa floresta, bastante vulnerável à pressão antrópica e à ocorrência de incêndios que ameaçam sua existência (BARETTA et al., 2008) os quais podem provocar desequilíbrios na comunidade de invertebrados, influenciando sua abundância e diversidade. Paisagens de floresta primária com Araucária e/ou secundária em estádio conservado podem contribuir para conservar a diversidade geral em agroecossistemas, bem como servir como abrigo para espécies de artrópodes (MOLINA; POGGIO; GHERSA, 2014). Ponge et al. (2013) mostram que a fauna do solo (exceto colêmbolos epígeos) e a comunidade microbiana são negativamente afetadas pelo aumento da intensificação agrícola, evidenciando a importância da manutenção de áreas de floresta para a conservação da biodiversidade da fauna do solo.

Como indicadores da qualidade do solo, a abundância e diversidade da fauna edáfica, integrados às propriedades microbiológicas, físicas e químicas do solo, podem melhor refletir a mudança ecológica geral do ecossistema em estudo (ASPETTI et al., 2010; VASCONCELLOS et al., 2013; PONGE et al., 2015). Nesse sentido, a abundância e diversidade desses organismos e/ ou a presença de grupos específicos, tais como formigas, aranhas, minhocas e colêmbolos, entre outros, podem ser utilizados para caracterizar um determinado ecossistema como, por exemplo, florestas naturais com araucária e áreas de reflorestamento (BARETTA et al., 2008; BARETTA; BROWN; CARDOSO, 2010; PEREIRA et al., 2017).

A fauna edáfica é um componente importante do ecossistema terrestre porque contribui com processos ecológicos (ROŹEN et al., 2010), principalmente atuando em sinergismo com a microbiota do solo, na decomposição da matéria orgânica e ciclagem de nutrientes, beneficiando 
a aeração do solo, infiltração de água e funcionamento biológico do solo (LAVELLE; SPAIN, 2001). A intensa interação da diversificada comunidade de invertebrados edáficos com os demais atributos físicos, químicos e microbiológicos do solo, torna-os importantes indicadores de qualidade, principalmente por sua rápida resposta às alterações do meio, em comparação aos atributos físicos e químicos do solo (BARETTA; BROWN; CARDOSO, 2010; ROUSSEAU et al., 2013). Dessa forma, a manutenção da biodiversidade do solo é fundamental para a sua qualidade (CROTTY et al., 2015).

A relevância ecológica, econômica e paisagística da Floresta Ombrófila Mista, além da extrema vulnerabilidade à extinção da A. angustifolia (BROCARDO; CÂNDIDO JÚNIOR, 2012) indica a urgente necessidade de estudos que revelem mais detalhadamente a relação entre a comunidade de organismos da fauna do solo e sua participação nos processos ecológicos importantes na conservação dessa floresta. A hipótese do estudo é que o impacto antrópico na Floresta de Araucária pode interferir na abundância e diversidade da fauna do solo e que as variáveis ambientais, ou seja, as propriedades químicas, físicas e microbiológicas do solo ajudam a explicar a distribuição da fauna edáfica. Nesse contexto, este trabalho tem como objetivo avaliar o potencial da fauna edáfica e das variáveis ambientais explicativas na discriminação entre as florestas com araucária nativa e reflorestada, a fim de selecionar indicadores de qualidade do solo.

\section{Material e métodos}

\section{Descrição das áreas de estudo}

O estudo foi realizado em três regiões de Floresta Ombrófila Mista, pertencente ao Bioma Mata Atlântica, no estado de São Paulo. Parcelas com 0,5-1,0 ha foram selecionadas em áreas de floresta nativa (NF) e reflorestada (RF) com A. angustifolia. O clima da região é subtropical úmido, sem estação seca e verões amenos $(\mathrm{Cfb})$, de acordo com Köppen. As áreas de floresta nativa têm acima de 120 anos de idade e as áreas de reflorestamento apresentam idade de plantio entre 20 e 45 anos. As áreas de cada região foram consideradas réplicas verdadeiras por serem representativas das áreas de florestas da região e suas características estão descritas na Tabela 1.

\section{Delineamento experimental e amostragem}

As coletas foram realizadas no inverno (agosto de 2009) e no verão (fevereiro de 2010). Em cada área, foram selecionadas, ao acaso, 15 árvores de araucária, com espaçamento mínimo de $20 \mathrm{~m}$. A dois metros do tronco de cada árvore, coletaram-se amostras de solo para análises microbiológicas, químicas e físicas e da serapilheira para análise química e de matéria seca.

Para as avaliações dos atributos microbiológicos, químicos e físicos, retiraram-se cinco subamostras de solo, em cada ponto de coleta (árvore), com auxílio de um trado holandês, na camada de $0-20 \mathrm{~cm}$, perfazendo 15 amostras compostas. A serapilheira foi amostrada, no mesmo ponto, numa área de $25 \times 25 \mathrm{~cm}$.

Para a análise microbiológica, as amostras foram peneiradas (malha de $2 \mathrm{~mm}$ ) e mantidas sob refrigeração $\left(4^{\circ} \mathrm{C}\right)$. Para a análise química, as amostras foram secas ao ar, peneiradas $(2 \mathrm{~mm})$ e armazenadas à temperatura ambiente. Para a análise física, foram coletadas amostras deformadas e indeformadas. Amostras deformadas foram secas ao ar, peneiradas $(2 \mathrm{~mm})$ e armazenadas. No inverno, nos mesmos pontos coletados, foram retiradas amostras indeformadas, usando anéis de aço inoxidável com diâmetro de $5 \mathrm{~cm}$, as quais foram mantidas intactas e seladas (para evitar a perda de umidade). 
Tabela 1 - Descrição das regiões e das áreas de coleta, modificado de Pereira et al. (2013)

Table 1 - Description of regions and samplings areas, modified from Pereira et al. (2013)

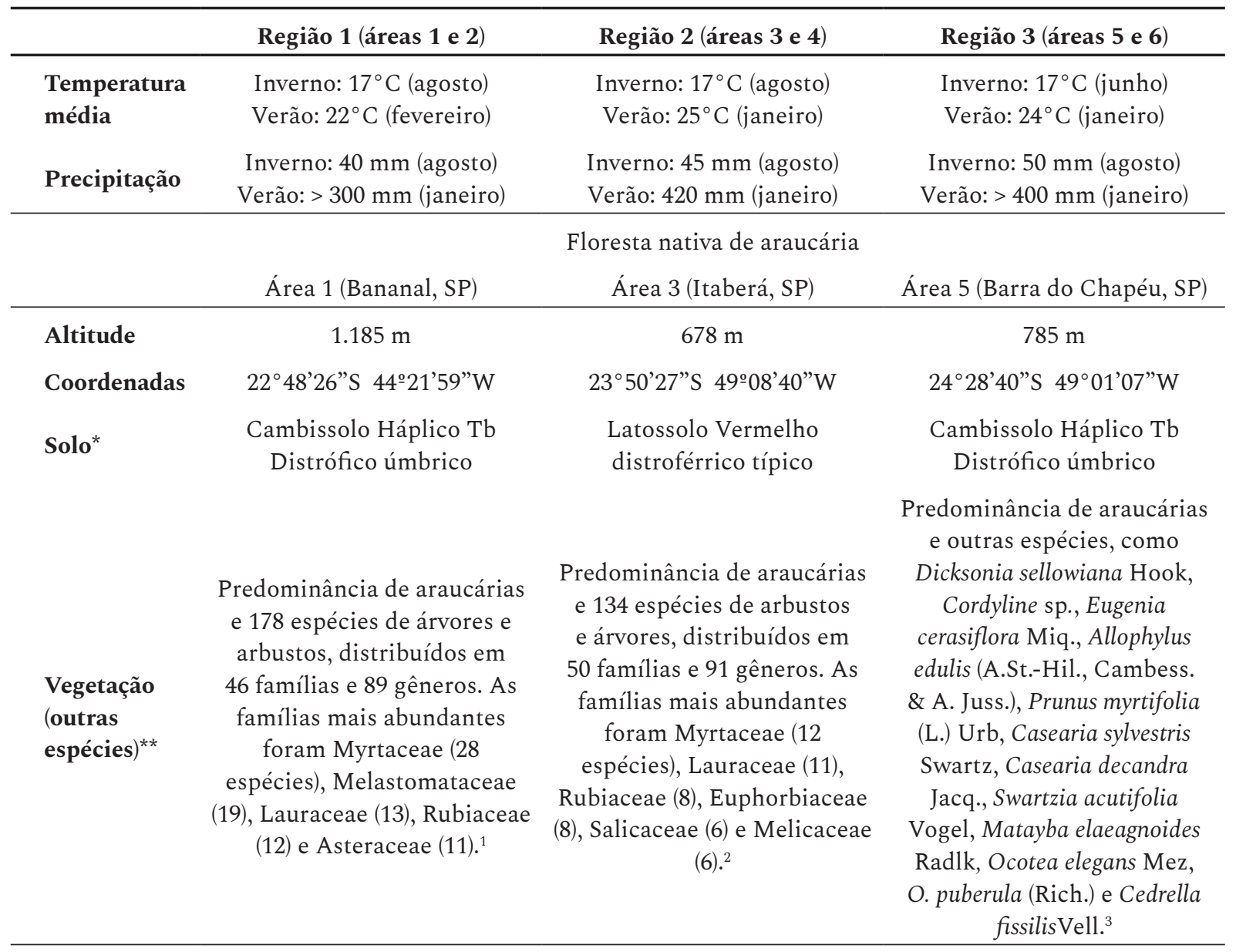

Reflorestamento de araucária

\begin{tabular}{|c|c|c|c|}
\hline & Área 2 (Bananal, SP) & Área 4 (Itapeva, SP) & Área 6 (Iporanga, SP/PETAR) \\
\hline Altitude & $1.126 \mathrm{~m}$ & $740 \mathrm{~m}$ & $932 \mathrm{~m}$ \\
\hline Coordenadas & $22^{\circ} 48^{\prime} 24^{\prime \prime} \mathrm{S} 44^{\circ} 22^{\prime} 27^{\prime \prime} \mathrm{W}$ & $24^{\circ} 04^{\prime} 19^{\prime \prime} \mathrm{S} 49^{\circ} 04^{\prime} 10^{\prime \prime} \mathrm{W}$ & $24^{\circ} 20^{\prime} 14^{\prime \prime} \mathrm{S} 48^{\circ} 36^{\prime} 14^{\prime \prime} \mathrm{W}$ \\
\hline Solo & $\begin{array}{l}\text { Cambissolo Háplico } \mathrm{Tb} \\
\text { Distrófico úmbrico }\end{array}$ & $\begin{array}{l}\text { Nitossolo Vermelho } \\
\text { Eutroférrico latossólico }\end{array}$ & $\begin{array}{l}\text { Cambissolo Háplico } \mathrm{Tb} \\
\text { Distrófico úmbrico }\end{array}$ \\
\hline $\begin{array}{l}\text { Vegetação } \\
\text { (outras } \\
\text { espécies) }\end{array}$ & $\begin{array}{l}\text { Ocorrência de araucárias } \\
\text { e ouras espécies, como D. } \\
\text { sellowiana, Euterpe edulis } \\
\text { Mart., Myrcia rostrata DC., } \\
\text { entre outras, herbáceas e } \\
\text { arbustos e gramíneas em } \\
\text { menor densidade. }\end{array}$ & $\begin{array}{l}\text { Predominância de araucárias } \\
\text { e espécies de sub-bosque, } \\
\text { como Gochnatia polymorpha } \\
\text { (Less) Cabr., Chusquea } \\
\text { ramosissima Lindm. }\end{array}$ & $\begin{array}{c}\text { Predominância de araucárias } \\
\text { e espécies de árvores } \\
\text { regenerativas, arbustos } \\
\text { e gramíneas (em menor } \\
\text { densidade) crescem nesta } \\
\text { área. }\end{array}$ \\
\hline
\end{tabular}

Fonte: Autores (2020)

Em que: *Classificação do solo de acordo com Embrapa (2006). ${ }^{* *}$ A composição florística está descrita com mais detalhes em Santos et al. (2009) ${ }^{1}$, Santos e Ivanauskas $(2010)^{2}$ e Souza $(2008)^{3}$. 


\section{Fauna edáfica}

Para a avaliação da atividade da fauna edáfica foram instaladas ao acaso 15 armadilhas de queda (Pitfall traps) próximas às árvores. Cada armadilha era constituída de um frasco de vidro de $6 \mathrm{~cm}$ de diâmetro e $12 \mathrm{~cm}$ de altura, o qual foi enterrado no solo, com a extremidade superior aberta e nivelada com a superfície do mesmo, permanecendo na área por três dias. Cada armadilha recebeu $200 \mathrm{~mL}$ de solução detergente neutra, na concentração de $2,5 \%$. Os invertebrados coletados em cada armadilha foram preservados em solução de álcool $75 \%$ até sua identificação em grandes grupos taxonômicos, com auxílio de microscópio estereoscópico e literatura especializada (GALLO et al., 2002; HUTCHINS et al., 2003a; 2003b). A pós a identificação, foram determinados a abundância (indivíduos por armadilha, ind. arm. ${ }^{-1}$ ), riqueza e os índice de diversidade de Shannon (H), de dominância de Simpson (Is) e de uniformidade de Pielou (e).

\section{Parâmetros microbiológicos}

Para as análises microbiológicas, amostras de $100 \mathrm{~g}$ de solo, com umidade corrigida para $60 \%$ da capacidade máxima de retenção de água, foram usadas para quantificar o carbono da biomassa microbiana (CBM) (VANCE; BROOKES; JENKINSON, 1987), sendo utilizado o valor de 0,33 como fator de correção dos cálculos. A atividade microbiana foi avaliada pela determinação da respiração basal (C-CO ${ }_{2}$ liberado) (ALEF; NANNIPIERI, 1995). Foram calculados o quociente metabólico $\left(q \mathrm{CO}_{2}\right)$ e o quociente microbiano ( $q \mathrm{Mic}$ ) (ANDERSON; DOMSCH, 1993).

A enzima desidrogenase (Desi) é muito ativa em células vivas e reflete a capacidade oxidativa da biomassa microbiana do solo. Para quantificar a atividade da desidrogenase, amostras de solo (5 g na umidade natural) foram usadas, adicionando $5 \mathrm{~mL}$ de solução de cloreto de trifeniltetrazólio $1,0 \%$ (TTC), incubando a $37{ }^{\circ} \mathrm{C}$ por $24 \mathrm{~h}$. O TTC utilizado como aceptor de elétrons, a partir da ação da enzima, é reduzido a trifeniltetrazólio formazan (TTF) sendo extraído com $10 \mathrm{~mL}$ de metanol. A atividade da enzima foi medida espectrofotometricamente a 485 nm (CASIDA JUNIOR; KLEIN; SANTORO, 1964).

\section{Parâmetros químicos e físicos}

Os parâmetros químicos do solo analisados foram $\mathrm{pH}\left(\mathrm{CaCl}_{2}\right)$, cátions trocáveis $\left(\mathrm{Al}^{3+}, \mathrm{Ca}^{2+}\right.$, $\mathrm{Mg}^{2+}$ e $\mathrm{K}^{+}$), $\mathrm{P}, \mathrm{H}+\mathrm{Al}$ e teor de carbono orgânico (C-org) (VAN RAIJ et al., 2001). Nas amostras deformadas, os seguintes parâmetros físicos do solo foram analisados: granulometria (GEE; OR, 2002) e umidade do solo (EMBRAPA, 2011). Nas amostras indeformadas de solo foram determinadas a densidade do solo (Ds) (BLAKE; HARTGE, 1986), a porosidade total (Pt), a microporosidade (Mi) e a macroporosidade (Ma) (EMBRAPA, 2011).

\section{Matéria seca, $\mathrm{C}, \mathrm{N}$ e $\mathrm{S}$ da serapilheira}

A matéria seca da serapilheira foi determinada a $55^{\circ} \mathrm{C}$ até massa constante. Em seguida, a serapilheira foi moída, passada em peneira de 100 mesh e o teor de C, N e S foi determinado por combustão seca em analisador simultâneo para C, N, S, H, modelo vario EL cube, marca Elementar.

\section{Análise estatística}

A abundância, riqueza e os índices de diversidade de Shannon, de dominância de Simpson e de uniformidade de Pielou, foram submetidos à análise de variância (ANOVA Two-way) e as médias comparadas pelo teste LSD ( $\mathrm{p} \leq 0,05)$ por meio do programa estatístico SAS (SAS INSTITUTE, 2002). 
Os dados de abundância de morfotipos foram submetidos à Análise de Componentes Principais (ACP), para as épocas estudadas (inverno e verão), utilizando o software CANOCO 4.0 (TER BRAAK; ŠMILAUER, 2002). Somente os atributos químicos do solo ( $\mathrm{pH} \mathrm{CaCl}, \mathrm{P}, \mathrm{Ca}, \mathrm{K}$ e C-org), físicos [densidade (Ds), macroporosidade (Ma), porosidade total (Pt) e umidade do solo (Umi)], os da serapilheira [carbono (C-ser) e nitrogênio (N-ser)], além de massa seca de serapilheira (MSS) e os microbiológicos do solo [(CBM e Desidrogenase (Desi)], além da riqueza (Riq) e abundância (Abun) foram significativos $(\mathrm{p}<0,05)$ e utilizados como variáveis ambientais explicativas, aumentando-se assim a robustez da análise.

Adicionalmente, as variáveis ambientais e os principais atributos da fauna foram submetidos à Análise Canônica Discriminante (ACD) para identificar quais deles foram mais relevantes na separação das áreas (BARETTA etal., 2005). Foram calculados os Coeficientes Canônicos Padronizados (CCP), o Coeficiente de Correlação $(r)$ e Taxa de Discriminação Paralela (TDP). Os valores de TDP indicam a força da variável em discriminar as áreas estudadas nas quais os valores são considerados bons (entre 0,1 e 0,2), muito bons (entre 0,2 e 0,4), ótimos (entre 0,4 e 0,8) e excelentes (acima de 0,81). Valores positivos indicam efeitos de separação entre a floresta nativa e reflorestada, enquanto que os negativos indicam semelhanças do atributo entre as florestas nativa e reflorestada (BARETTA; BROWN; CARDOSO, 2010; PEREIRA et al., 2017).

\section{Resultados e discussão}

\section{Abundância e diversidade da fauna edáfica}

Coletaram-se um total de 2.944 indivíduos da fauna edáfica (1.481 ind. na área NF e 1.463 ind. em RF) no inverno e 5.322 indivíduos (3.282 ind. em NF e 2.040 ind. em RF) no verão, indicando certa homogeneidade entre as florestas em escala regional. Entre a fauna edáfica, no inverno, o grupo Collembola foi o mais abundante, com $29,3 \%$ da abundância total (ind. arm. ${ }^{-1}$ ), enquanto Formicidae representou $26,9 \%$ e Coleoptera $23,7 \%$. No verão, à semelhança do inverno, destacou-se a abundância do grupo Collembola com $54,5 \%$, seguido por Formicidae com $25,3 \%$ da abundância total. Qualquer distúrbio ou estresse imposto a um ecossistema interage com fatores ambientais, que podem variar com o tempo e espaço. Assim, diferentes fatores influenciaram as populações da fauna edáfica nas áreas de araucárias, como estabilidade do ecossistema (por exemplo, o tempo já decorrido de plantio, acima de 20 anos, das áreas reflorestadas) e fatores climáticos (COYLE et al., 2017).

Os valores médios de abundância no inverno (33 ind. arm. $\left.{ }^{-1}\right)$ não diferiram entre NF e RF. Já no verão, foram maiores $(\mathrm{p}<0,05)$ em NF $\left(73\right.$ ind. $\left.\operatorname{arm} .^{-1}\right)$ em comparação com RF (45 ind. arm. $\left.{ }^{-1}\right)$. Diferenças entre as épocas de amostragens $(\mathrm{p}<0,05)$ também aconteceram nas duas áreas (Figura 1). Variações de abundância ocorreram ao longo do tempo entre as áreas NF e RF, com um aumento em densidades populacionais durante o verão (Figura 1), quando a precipitação e a temperatura foram altas e favoráveis para uma maior atividade da fauna edáfica (Tabela 1). Esse resultado mostra que essa maior abundância provavelmente contribua para a melhoria do funcionamento biológico do solo (BRUSSAARD; RUITER; BROWN, 2007) e, principalmente, para a manutenção do equilíbrio biológico e melhoria da estrutura da cadeia alimentar (ROUSSEAU et al., 2013). No entanto, mesmo que esses fatores possam influenciar e causar flutuações nas populações da fauna edáfica, as diferenças de riqueza e índices de diversidade entre as áreas são bastante estáveis (Figura 1), o que pode indicar que está ocorrendo uma estabilidade da comunidade edáfica e das áreas reflorestadas comparável às nativas. Alguns estudos (JING et al., 2005; MAUNSELL et al., 2013; MUMLADZE et al., 2015; RÖDER et al., 2017) têm apontado o efeito da altitude na densidade e diversidade de organismos da fauna edáfica, como, por exemplo, alguns insetos, ácaros e colêmbolos. Embora não tenha sido explorado especificamente, as áreas ficam em regiões de Mata Atlântica, onde a altitude pode ser um fator que explique a diferença de abundância no verão, principalmente porque nessa época a maior frequência de chuvas em determinadas áreas, aliada a altas temperaturas do solo, permite maior atividade da fauna, principalmente estimulada pela busca de alimento e necessidade de reprodução. 
Algumas áreas RF apresentaram maiores altitudes, onde mudanças climáticas são consideradas um dos principais fatores relacionados ao gradiente de altitude (JING et al., 2005).

Figura 1 - Valores médios de abundância, riqueza e índices de diversidade de grupos taxonômicos da fauna edáfica nas Florestas de Araucária nativa e reflorestada no inverno e verão. Letra minúscula compara as florestas independentemente de época e letra maiúscula compara as florestas dentro de uma mesma época

Figure 1 - Average values of abundance, richness and diversity indexes of taxonomic groups of edaphic fauna in native and reforested Araucaria forests in winter and summer. Lowercase letters compare forests regardless of the season and capital letters compare the forests within the same season

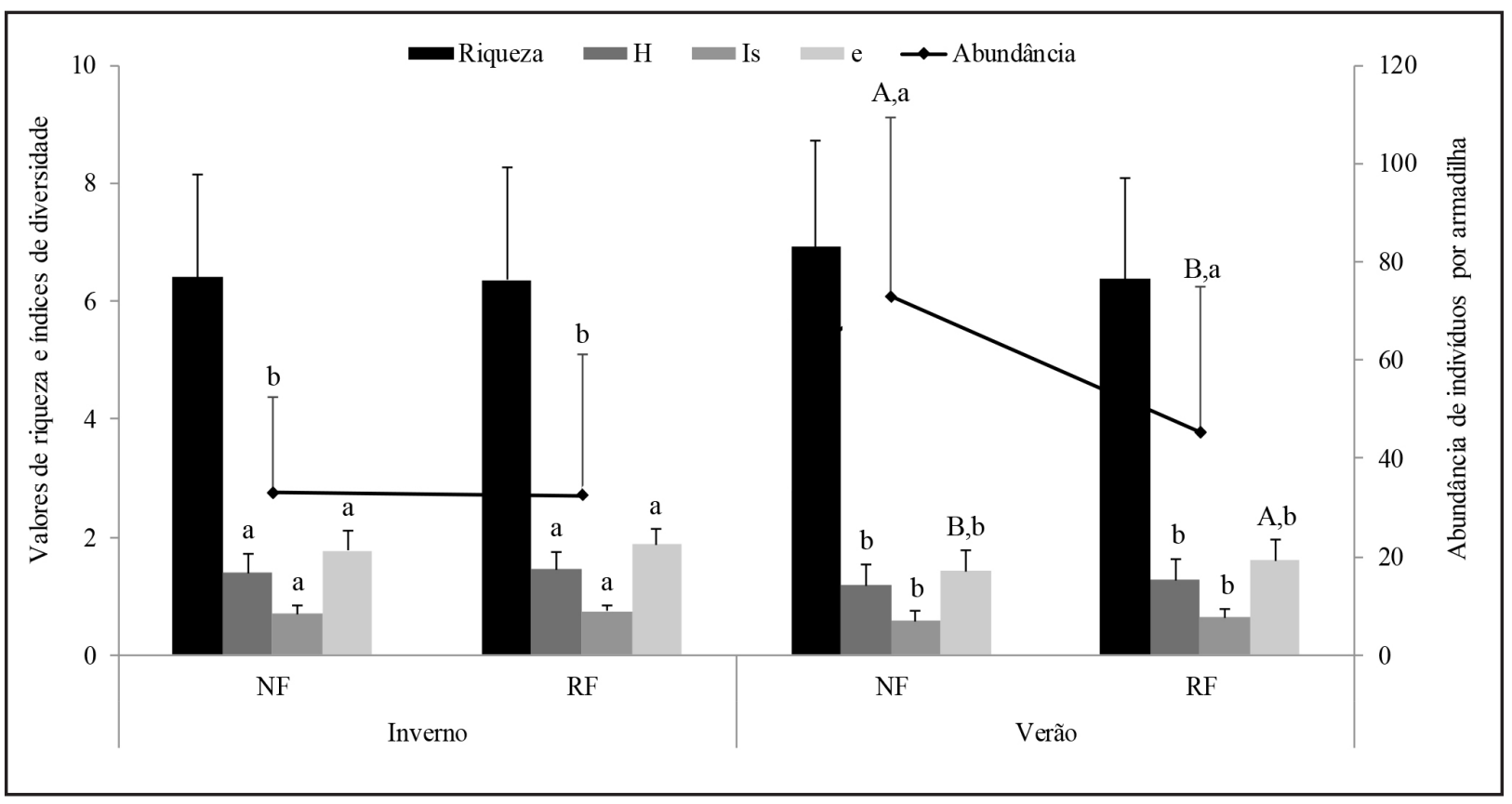

Fonte: Autores (2020)

Em que: $\mathrm{H}$ = Diversidade de Shannon; Is = Dominância de Simpson; $e=$ Uniformidade de Pielou; NF = Florestas de Araucária nativa; $\mathrm{RF}=$ Araucária reflorestada. A ausência de letra indica que não houve diferença significativa entre florestas e épocas pelo teste de LSD $(\mathrm{p} \leq 0,05)$.

A riqueza da fauna edáfica não diferiu entre os tratamentos e épocas de amostragem, indicando que a atividade da fauna edáfica não foi sensível ao efeito de sazonalidade e nem respondeu à maior oferta de recurso alimentar encontrado em solos sob floresta mais preservada, como é o caso da NF (Figura 1). Em outro trabalho (PEREIRA et al., 2017), avaliando a macrofauna edáfica das mesmas áreas, os autores constataram diferenças na riqueza, sendo maior na NF e no verão, sendo atribuído às melhores condições climáticas e de solo (heterogeneidade e quantidade de serapilheira).

Os maiores valores dos índices de diversidade foram encontrados em NF e RF no inverno, quando comparados ao verão. Por outro lado, o índice de Pielou, que reflete a uniformidade da distribuição da fauna edáfica em cada área, foi maior em RF no verão em comparação a NF (Figura 1). Os dados indicam que os dois ambientes de araucária suportaram uma abundância e diversidade da fauna edáfica muito semelhante (Figura 1), que foi numericamente dominada por Collembola, Formicidae e Coleoptera. Comparada à floresta nativa NF, a área RF é caracterizada pela manutenção de uma cobertura vegetal, embora menos diversificada e menos densa, inclusive, com cobertura de gramínea em locais mais abertos (Tabela 1). Isso provavelmente reflete a estabilidade do ecossistema 
devido à diversidade de plantas, dossel menos denso, sub-bosque em regeneração, cobertura de serapilheira e umidade do solo (BEEBY, 1993). Esses resultados podem ser decorrentes da estrutura de espécies vegetais formada há mais de 20 anos no reflorestamento, por apresentar um dossel relativamente uniforme no porte, com árvores emergentes, com solo permanentemente coberto por serapilheira e sem ação antrópica recente, o que, por sua vez, favorece a colonização e conservação da biodiversidade da fauna edáfica. Na Mata Atlântica, a regeneração da floresta faz parte de um intrincado e complexo processo de sucessão, envolvendo espécies pioneiras, intermediárias e tardias, as quais interagem com o clima, fertilidade do solo e proximidade com bancos de germoplasma. Nesse sentido, mudanças ambientais durante a sucessão aumentam a quantidade de recursos basais que fornecem vários recursos de micro-hábitat e nutrientes para artrópodes que poderiam levar ao estabelecimento de uma comunidade diversa (MAHARNING; MILLS; ADL, 2009). Assim, o aumento na quantidade de espaço habitável, criado pelo aumento da área da superfície dos poros, por exemplo, aumentaria a abundância dos artrópodes (BERG; HEMERIK, 2004). O que ainda não está claro é como diferentes grupos de organismos, como invertebrados edáficos, respondem à sucessão de plantas, bem como a resposta da comunidade e diversidade desses organismos pode não ser consistente com a sucessão de plantas (MENTA, 2012). Outro ponto a ressaltar, mesmo após os mais de 20 anos de reflorestamento, considerando a semelhança entre abundância e diversidade da fauna edáfica, entre as áreas com araucárias, é que não há garantia de que toda a diversidade original seja restaurada, especialmente para as espécies endêmicas que são mais sensíveis a perturbações (ZEPPELINI et al., 2009).

\section{Análise de Componentes Principais}

A ACP revelou que os dois primeiros eixos explicaram 34,2 e $32,2 \%$ da variabilidade total dos dados no inverno e verão, respectivamente (Figuras $2 \mathrm{~A}$ e $2 \mathrm{~B}$ ). Esses resultados mostram uma nítida separação entre NF e RF, nos quais a fauna edáfica e variáveis ambientais explicativas assumiram uma distribuição diferenciada entre NF e RF nas duas épocas de coleta (Figuras 2A e 2B). Devido à natureza muito complexa do solo e a necessidade, mais precisa, da avaliação de sua qualidade, faz-se uso de diferentes indicadores, principalmente envolvendo os atributos físicos, químicos e biológicos, além da interação entre eles (LIMA et al., 2013). As variáveis ambientais medidas no presente estudo são conhecidas por afetarem a abundância da fauna edáfica.

No inverno, observa-se que os grupos Hemiptera, Collembola e Diplopoda ficaram mais associados à RF devido, principalmente, aos maiores valores de MSS (Figura 2A). Já em NF, os grupos Orthoptera, Hymenoptera, Araneae, Coleoptera, além do grupo "Outros" foram os mais abundantes. Isso é explicado pela associação dos atributos do solo (CBM, C-org, Ca, K, Pt, Ma) e serapilheira (C-Ser, N-Ser) com NF (Figura 2A). No verão, a maior abundância de grupos ficou associada à NF, destacando-se os grupos Hymenoptera (com a maioria em Formicidae), Hemiptera, Orthoptera, Araneae, Coleoptera e Collembola (Figura 2B). A atividade desses grupos foi mais intensa em solo com maiores teores de CBM, Ca, P, C-org, macroporosidade e atividade de desidrogenase. O acúmulo de $\mathrm{C}$ no solo resulta na formação de uma camada de serapilheira, o que aumenta o espaço habitável para os organismos do solo. A decomposição do carbono orgânico é facilitada pela ação de Formicidae, Orthoptera, Coleoptera e Collembola, influenciando na ciclagem de nutrientes (Ca e P) e favorecendo a atividade de microrganismos, evidenciado pela associação com o CBM e a desidrogenase, além de sugerir uma interação entre organismos da fauna e microrganismos do solo. Ainda, a ação de Formicidae, Orthoptera, Coleoptera cria canais no solo (macroporosidade) influenciando o transporte de água e gases no solo, modificando também o micro-hábitat de outros organismos, como Araneae e Collembola. Nenhum táxon da fauna edáfica ficou, especificamente, associado com a RF, resultando em menor abundância, embora o solo, em RF, apresentasse maiores valores de umidade, MSS e densidade (Figura 2B). Os grupos Orthoptera, Araneae e Hymenoptera, associados à NF, nas duas épocas e Formicidae, no inverno (Figuras $2 \mathrm{~A}$ e 2B), relacionaram-se a solos com valores mais altos de CBM. 
Figura 2 - Relação entre a Componente Principal 1 e 2, discriminando Florestas de Araucária nativa e reflorestada, grupos taxonômicos da fauna edáfica (em itálico) e as variáveis ambientais explicativas $(\rightarrow$ pontilhada) coletadas com armadilhas de queda no inverno e verão

Figure 2 - Relation between Principal Components 1 and 2, discriminating native Araucaria forests from reforested ones, taxonomic groups (in italics) and explanatory environmental variables $(\rightarrow$ dotted), collected with pitfall traps in winter and summer

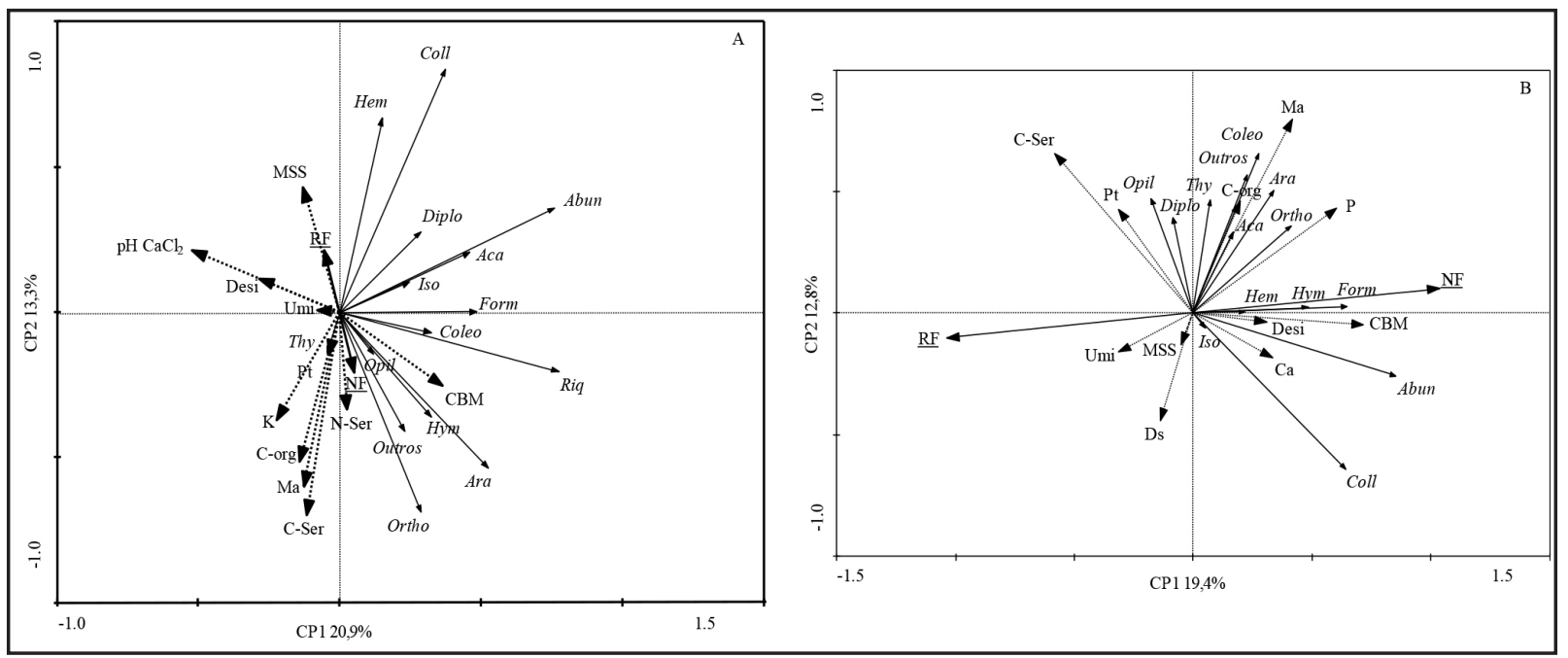

Fonte: Autores (2020)

Em que: $\mathrm{CP}=$ Componente Principal; NF = Florestas de Araucária nativa; $\mathrm{RF}=$ Araucária reflorestada; $\mathrm{A}=\mathrm{Inverno}$; $\mathrm{B}=$ Verão; Abun = Abundância Aca = Acarina; Ara = Araneae: Coleo $=$ Coleoptera Coll $=$ Collembola; Diplo $=$ Diplopoda; Form = Formicidae; Hem = Hemiptera; Hym = Hymenoptera; Iso = Isopoda; Opil = Opilionidae; Ortho = Orthoptera; Outros = Outros grupos menos abundantes; Riq = Riqueza; Thy = Thysanoptera. $\mathrm{CBM}=\mathrm{Carbono}$ da biomassa microbiana; Desi = Enzima desidrogenase; $\mathrm{C}$-org = Carbono orgânico; $\mathrm{Ca}=\mathrm{Cálcio} ; \mathrm{K}=$ Potássio; $\mathrm{P}=$ Fósforo; Umi = Umidade do solo; $\mathrm{Ds}=$ Densidade do solo $; \mathrm{Pt}=$ Porosidade total; $\mathrm{Ma}=$ Macroporosidade MSS = Matéria seca da serapilheira; C-Ser = Carbono da serapilheira; N-Ser = Nitrogênio da serapilheira.

Evidenciou-se que a melhor cobertura vegetal, considerada de melhor qualidade em NF, beneficiou a comunidade da fauna edáfica (MARTIUS et al., 2004; BARETTA; BROWN; CARDOSO, 2010), pois proporcionou maior quantidade de $\mathrm{C}$ e $\mathrm{N}$ na serapilheira e ao solo maior quantidade de $\mathrm{P}$ e Ca, elementos chaves ao desenvolvimento da fauna (BARETTA et al., 2011), além de aumento no $\mathrm{CBM}$ e, consequentemente, maior estímulo à microbiota (Figuras 2A e 2B). Mesmo sendo considerada de menor qualidade por não ter apresentado relação com nutrientes, o reflorestamento com araucária correlacionou-se com a matéria seca da serapilheira (Figuras 2A e 2B). Dessa forma, proporcionou modificações estruturais do hábitat em decorrência da presença de outras espécies vegetais nas áreas favorecendo a comunidade da fauna edáfica, especialmente pela maior umidade do solo, assemelhando-se à floresta nativa (Figuras 2A e 2B). Maior diversidade de espécies vegetais resulta em aumento na diversidade da comunidade de invertebrados (CONG et al., 2015), em decorrência da maior diversificação de micro-hábitats, além da maior complexidade estrutural do solo, oriunda da decomposição dos resíduos vegetais. Isso contribui para aumentar a diversidade de grupos funcionais (BABEL; EHRMANN; KREBS, 1992) e as mudanças nas comunidades da fauna durante a sucessão podem ser relacionadas, principalmente, com modificações na estrutura do hábitat (DECAËNS et al., 1998). O reflorestamento, do ponto de vista nutricional, não proporcionou um resultado similar à área NF, o que indica ser necessário um maior tempo para que as relações ambientais possam restabelecer a complexidade ecológica nos ecossistemas florestais. 


\section{Análise Canônica Discriminante (ACD)}

O teste estatístico multivariado de Wilks' Lambda para os atributos da fauna invertebrada e variáveis ambientais indicou diferenças significativas entre épocas de amostragem e Florestas de Araucária (floresta nativa e reflorestada) $(\mathrm{p}<0,0001)$ quanto à função canônica discriminante 1 (FCD1) e 2 (FCD2), sendo realizada uma ACD para cada época de amostragem (Figura 3). Ainda, foi observada alta correlação canônica para a época seca (inverno) $(0,92)$ e época chuvosa (verão) $(0,94)$. $O$ modelo utilizado da ACD explicou boa parte da variabilidade presente nas florestas. A distribuição polarizada das Florestas de Araucária (floresta nativa e reflorestada) indica alta dissimilaridade entre as áreas estudadas, em que a FCD1 separou a área NF da área RF (Figuras 3A e 3B).

Figura 3 - Coeficientes Canônicos Padronizados da Função Canônica Discriminante 1 e 2, discriminando Florestas de Araucária nativa e reflorestada, considerando os atributos das variáveis ambientais (químicas, físicas e microbiológicas do solo e química da serapilheira) e da fauna do solo coletada com armadilhas, no inverno e verão

Figure 3 - Standardized Canonical Coefficients of the Canonical Discriminant Functions 1 and 2, discriminating native Araucaria forests from the reforested ones, when considering properties of environmental variables (chemical, physical and microbiological characteristics of soil and litter chemistry) and of soil fauna collected with traps, in winter and summer
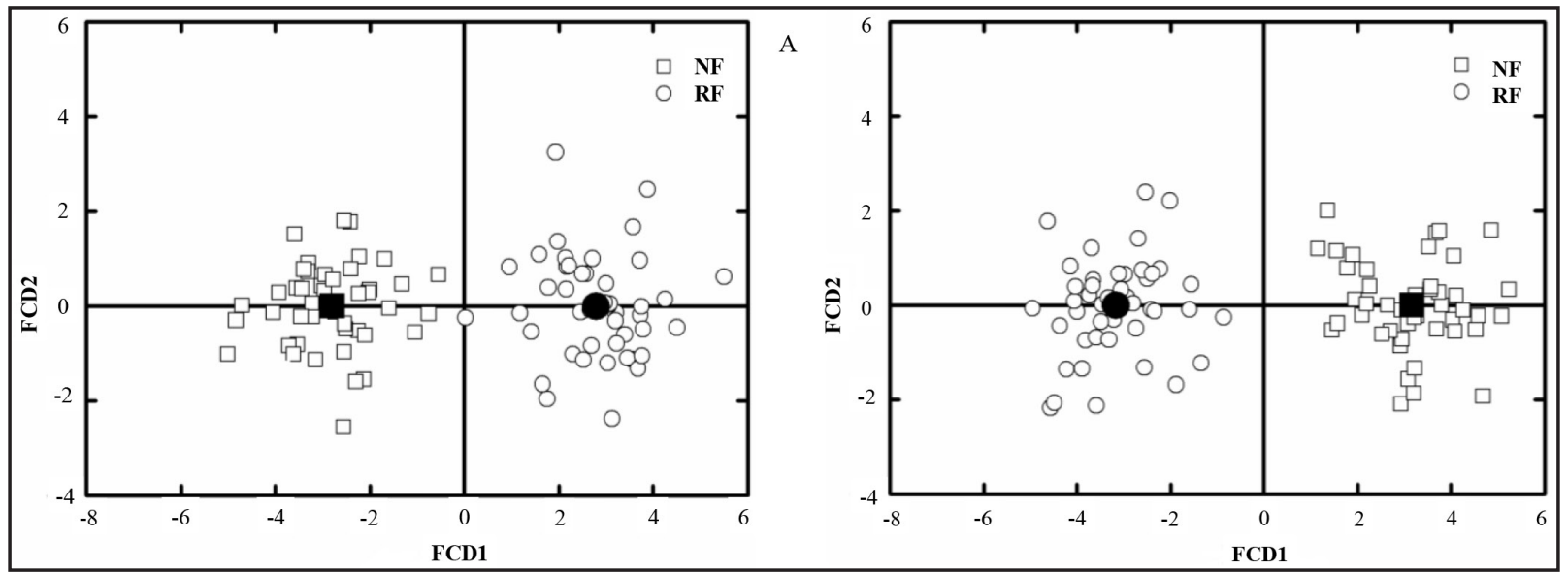

Fonte: Autores (2020)

Em que: CCP = Coeficientes Canônicos Padronizados; FCD = Função Canônica Discriminante; NF = Florestas de Araucária nativa; $\mathrm{RF}$ = Araucária reflorestada; A = Inverno; B = Verão. O símbolo cheio representa o valor médio de CCP para cada área $(n=45)$.

A Tabela 2 demonstra o potencial de cada atributo para discriminar a qualidade do solo, uma vez que contribuíram para a separação entre as florestas estudadas. No geral, os atributos foram considerados bons, (valores entre 0,1 e 0,2 ), com destaque para a umidade do solo, considerada muito bom (valores acima de 0,2 ), tanto no inverno quanto no verão.

Dentre os grupos da fauna, somente Orthoptera $(0,10)$ contribuiu para a separação das florestas no verão. A ordem Orthoptera é ecologicamente muito importante porque seus representantes, em sua maioria, são escavadores, detritívoros e herbívoros, beneficiados pelo ambiente encontrado nas duas áreas de araucária, destacando esse grupo como um importante indicador das modificações ambientais. Contudo, há necessidade de estudos mais aprofundados sobre esse grupo que inclui gafanhotos, grilos, esperanças e paquinhas. Outros atributos da fauna edáfica contribuíram para a separação das florestas, tais como a abundância $(0,20)$, no verão, e os 
índices de dominância de Simpson $(0,10)$ no inverno e uniformidade de Pielou $(0,12)$ no verão. Esses atributos são importantes, pois dão um indicativo da distribuição de grupos da fauna edáfica.

As variáveis ambientais consideradas indicadoras de mudanças nas florestas foram $\mathrm{P}(0,20)$, S-Ser $(0,12)$, Pt $(0,14)$, umidade do solo $(0,31)$, CBM $(0,10), \mathrm{C}-\mathrm{CO}_{2}(0,10)$ e desidrogenase $(0,10)$ no inverno; no verão as variáveis foram $\mathrm{pH} \mathrm{CaCl}_{2}(0,10), \mathrm{H}+\mathrm{Al}(0,11), \mathrm{P}(0,10), \mathrm{K}(0,10), \mathrm{S}-\mathrm{Ser}(0,10)$, Pt $(0,11)$, umidade do solo $(0,25)$ e CBM $(0,10)$. Os demais atributos das variáveis ambientais e da fauna foram menos sensíveis, assumindo menores valores da TDP e contribuíram menos na função indicada para a separação entre as florestas (Tabela 2). Os atributos químicos do solo (P, $\mathrm{K}, \mathrm{pH} \mathrm{CaCl}{ }_{2}$ e $\mathrm{H}+\mathrm{Al}$ ) e o enxofre na serapilheira (S-Ser), são os que podem influenciar na atividade biológica do solo. Os atributos físicos do solo (Pt e umidade do solo) estão relacionados à capacidade de oxigenação e retenção de umidade, características fundamentais para o desenvolvimento radicular e de organismos, favorecendo os processos químicos e biológicos do solo. Já, os atributos microbiológicos estão relacionados com o carbono do solo, tais como CBM e atividade microbiana $\left(\mathrm{C}-\mathrm{CO}_{2}\right.$ e desidrogenase), que pode indicar maiores perdas de carbono entre as áreas de araucária.

Tabela 2 - Valores dos Coeficientes Canônicos Padronizados, Correlação Canônica e Taxa de Discriminação Paralela referentes à Análise Canônica Discriminante quanto aos atributos da fauna e variáveis ambientais no inverno e verão, independentemente da floresta avaliada

Table 2 - Standardized Canonical Coefficients, Canonical Correlation, and Parallel Discrimination Rate related to Canonical Discriminant Analysis containing fauna and environmental attributes, in winter and summer, regardless of the site

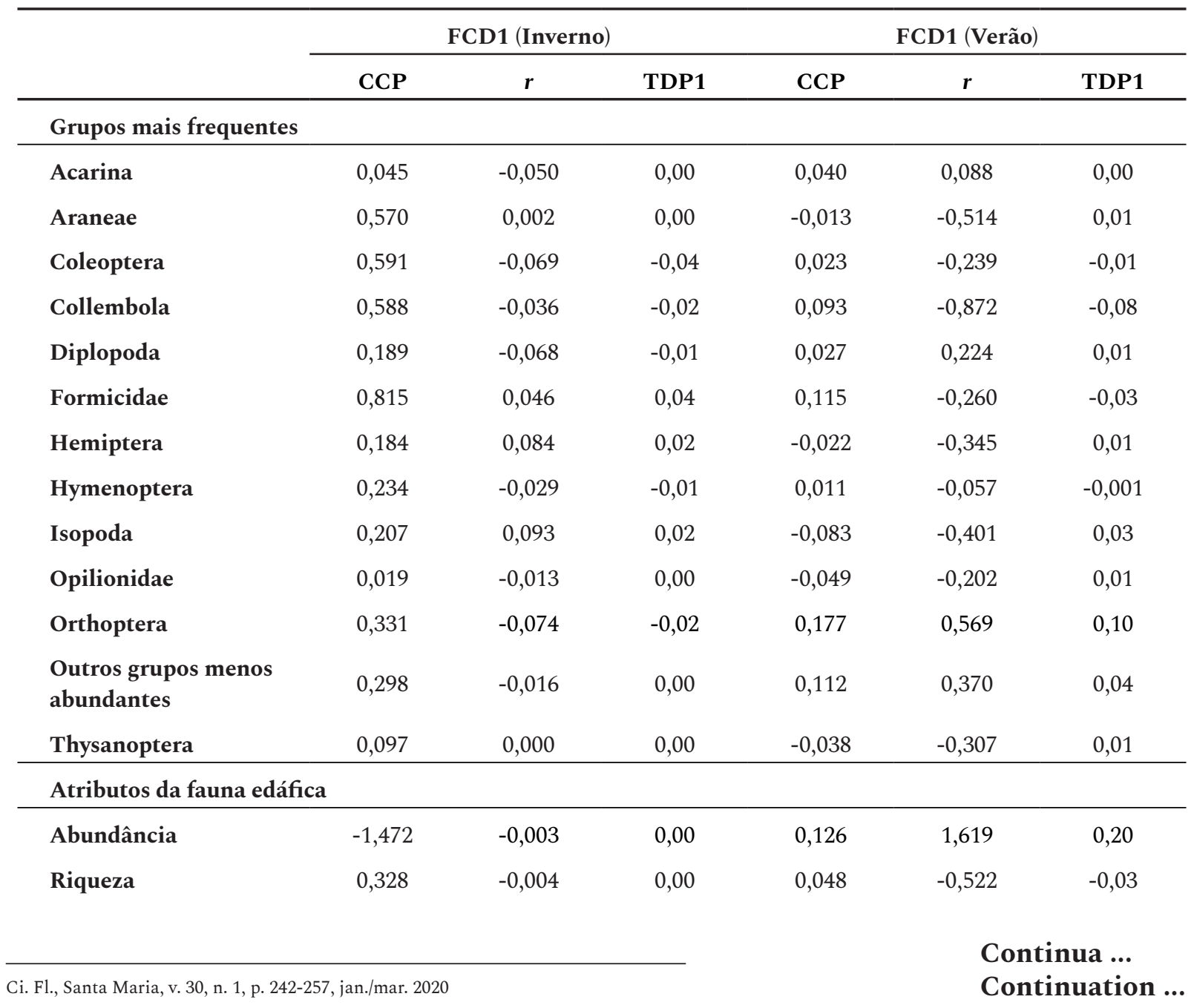


Tabela 2 - Conclusão ...

Table 2 - Conclusion ...

\begin{tabular}{|c|c|c|c|c|c|c|}
\hline & \multicolumn{3}{|c|}{ FCD1 (Inverno) } & \multicolumn{3}{|c|}{ FCD1 (Verão) } \\
\hline & CCP & $r$ & TDP1 & CCP & $r$ & TDP1 \\
\hline \multicolumn{7}{|l|}{ Atributos da fauna edáfica } \\
\hline $\begin{array}{l}\text { Diversidade de } \\
\text { Shannon }\end{array}$ & $-1,375$ & 0,030 & $-0,04$ & $-0,039$ & 0,484 & $-0,02$ \\
\hline $\begin{array}{l}\text { Dominância de } \\
\text { Simpson }\end{array}$ & 1,569 & 0,061 & 0,10 & $-0,054$ & 0,682 & $-0,04$ \\
\hline $\begin{array}{l}\text { Uniformidade de } \\
\text { Pielou }\end{array}$ & $-0,345$ & 0,058 & $-0,02$ & $-0,083$ & $-1,473$ & 0,12 \\
\hline \multicolumn{7}{|l|}{ Atributos } \\
\hline $\mathrm{pH} \mathrm{CaCl}{ }_{2}$ & 0,688 & 0,063 & 0,04 & $-0,029$ & $-1,861$ & 0,10 \\
\hline $\mathbf{H}+\mathbf{A l}$ & 1,018 & 0,043 & 0,04 & $-0,052$ & $-2,149$ & 0,11 \\
\hline Al & $-0,476$ & 0,025 & $-0,01$ & $-0,080$ & 1,100 & $-0,09$ \\
\hline $\mathbf{P}$ & $-1,140$ & $-0,178$ & 0,20 & 0,167 & 0,344 & 0,10 \\
\hline $\mathbf{K}$ & $-0,249$ & $-0,175$ & 0,04 & 0,102 & 0,544 & 0,10 \\
\hline Mg & $-0,070$ & $-0,014$ & 0,00 & 0,037 & $-0,351$ & $-0,01$ \\
\hline Carbono orgânico & $-0,164$ & 0,042 & $-0,01$ & $-0,018$ & 0,469 & $-0,01$ \\
\hline $\begin{array}{l}\text { Massa seca da } \\
\text { serapilheira }\end{array}$ & $-0,037$ & 0,005 & 0,00 & 0,078 & 0,224 & 0,02 \\
\hline $\begin{array}{l}\text { Carbono na } \\
\text { serapilheira }\end{array}$ & 0,757 & $-0,082$ & $-0,06$ & 0,077 & $-0,366$ & $-0,03$ \\
\hline $\begin{array}{l}\text { Nitrogênio na } \\
\text { serapilheira }\end{array}$ & $-0,158$ & $-0,274$ & 0,04 & 0,238 & 0,143 & 0,03 \\
\hline Enxofre na serapilheira & $-0,670$ & $-0,176$ & 0,12 & 0,163 & 0,411 & 0,10 \\
\hline \multicolumn{7}{|l|}{ Atributos físicos } \\
\hline Densidade do solo & 0,585 & $-0,061$ & $-0,04$ & 0,054 & $-0,947$ & $-0,05$ \\
\hline Porosidade total & 0,729 & 0,191 & 0,14 & $-0,168$ & $-0,654$ & 0,11 \\
\hline Macroporosidade & $-0,390$ & $-0,117$ & 0,05 & 0,103 & 0,234 & 0,02 \\
\hline Umidade do solo & 0,860 & 0,362 & 0,31 & $-0,227$ & $-1,119$ & 0,25 \\
\hline \multicolumn{7}{|l|}{ Atributos microbiológicos } \\
\hline $\begin{array}{l}\text { Carbono da biomassa } \\
\text { microbiana }\end{array}$ & $-1,009$ & $-0,059$ & 0,10 & 0,110 & 0,620 & 0,10 \\
\hline Respiração basal & 1,076 & 0,073 & 0,10 & $-0,022$ & $-1,000$ & 0,02 \\
\hline Quociente metabólico & $-0,432$ & 0,168 & $-0,07$ & $-0,150$ & 0,170 & $-0,03$ \\
\hline Desidrogenase & 0,450 & 0,133 & 0,10 & $-0,066$ & 0,176 & $-0,01$ \\
\hline
\end{tabular}

Fonte: Autores (2020)

Em que: $\mathrm{FCD}=$ Função Canônica Discriminante; $r=$ Correlação Canônica; $\mathrm{CCP}=$ Coeficientes Canônicos Padronizados; TDP = Taxa de Discriminação Paralela. Valores em negrito representam os atributos mais importantes para discriminar as áreas de estudo $(\mathrm{n}=45)$. 
Apesar da eficiência em separar as florestas, promovida pela análise canônica discriminante, a dissimilaridade entre as áreas de araucárias ocorreu principalmente em função dos atributos ambientais avaliados. Isso ocorreu porque os valores de TDP para fauna edáfica foram menores e contribuíram pouco na função indicada de separação entre as florestas (Tabela 2). Dessa forma, para uma avaliação mais eficiente da qualidade do solo, em florestas com araucárias, há a necessidade do uso de um conjunto de indicadores (químicos, físicos e biológicos), visto que todos desenvolvem um importante papel nesse ecossistema (BARETTA et al., 2007a; 2007b; 2008; MOREIRA et al., 2009; BARETTA; BROWN; CARDOSO, 2010; PEREIRA et al., 2013; 2017).

Este estudo é uma referência inicial da qualidade do solo dessas Florestas de Araucárias e pode ser utilizado para comparar os resultados futuros de como essas importantes florestas têm se mantidas conservadas. Entretanto, é importante mencionar que existe uma forte indicação de que as áreas que haviam sido cortadas e posteriormente voltaram a ser reflorestadas já apresentam uma estabilidade ambiental semelhante às áreas de florestas nativas, o que não deixa de ser um resultado muito promissor para aumentar a expectativa de que se possa proteger da extinção a $A$. angustifolia, sendo que atualmente ela se encontra sob tal ameaça.

\section{Conclusões}

As áreas de Floresta de Araucária nativa e reflorestada são semelhantes em relação à diversidade, riqueza e índices de grupos da fauna, os quais se mantêm estáveis entre florestas e época de coleta. O estado de conservação da Floresta de Araucária e os atributos químicos, físicos e microbiológicos do solo foram fatores determinantes nas condições ambientais que influenciaram a distribuição dos grupos de fauna edáfica nas áreas de florestas, onde a maioria dos grupos foi associada à floresta nativa que apresentou maior complexidade florística.

Os atributos do solo, como carbono da biomassa microbiana, atividade da enzima desidrogenase, carbono orgânico, cálcio, potássio, fósforo, umidade do solo, densidade do solo, porosidade total, macroporosidade, matéria seca da serapilheira, carbono da serapilheira e nitrogênio da serapilheira, foram determinantes possivelmente por condicionarem distribuição e/ou estabelecimento da fauna edáfica na floresta nativa e reflorestada.

\section{Agradecimentos}

À Fundação de Amparo à Pesquisa do Estado de São Paulo (FAPESP) pelo apoio à pesquisa (projeto no 2007/06981-2 do Programa Biota e projeto n 01/05146-6). EJBNC agradece por uma bolsa de produtividade do Conselho Nacional de Desenvolvimento Científico e Tecnológico (CNPq - 305193/2016-3). DB agradece ao CNPq pela Bolsa de Produtividade em Pesquisa (305939/2018-1). LCIOF é bolsista do CNPq - Brasil (155778/2018-8).

\section{Referências}

ALEF, K.; NANNIPIERI, P. Methods in apllied soil microbiology and biochemistry. London: Academic Press, 1995. 576 p.

ANDERSON, T. H.; DOMSCH, K. H. The metabolic quotient for $\mathrm{CO}_{2}\left(q \mathrm{CO}_{2}\right)$ as a specific activity parameter to assess the effects of environmental conditions, such as $\mathrm{pH}$, on the microbial biomass 
of forest soils. Soil Biology and Biochemistry, Oxford, v. 25, p. 393-395, 1993.

ASPETTI, G. P. et al. Assessment of soil-quality index based on microarthropods in corn cultivation in Northern Italy. Ecological Indicators, [s. l.], v. 10, n. 2, p. 129-135, 2010.

BABEL, U.; EHRMANN, O.; KREBS, M. Relationships between earthworms and some plant species in a meadow. Soil Biology and Biochemistry, Oxford, v. 24, n. 12, p. 1477-1481, 1992.

BARETTA, D.; BROWN, G. G.; CARDOSO, E. J. B. N. Potencial da macrofauna e outras variáveis edáficas como indicadores da qualidade do solo em áreas com Araucaria angustifolia. Acta Zoologica Mexicana, Xalapa, v. 26, p. 135-150, 2010.

BARETTA, D. et al. Colêmbolos (Hexapoda: Collembola) como bioindicadores de qualidade do solo em áreas com Araucaria angustifolia. Revista Brasileira de Ciência do Solo, Viçosa, v. 32, p. 2693-2699, 2008.

BARETTA, D. et al. Earthworm populations sampled using collection methods in atlantic forests with Araucaria angustifolia. Scientia Agricola, Piracicaba, v. 64, n. 4, p. 384-392, 2007b.

BARETTA, D. et al. Efeito do monocultivo de Pinus e da queima do campo nativo em atributos biológicos do solo no Planalto sul Catarinense. Revista Brasileira de Ciência do Solo, Viçosa, v. 29, n. 5, p. 715-724, 2005.

BARETTA, D. et al. Fauna edáfica e qualidade do solo. In: KLAUBERG-FLHO, O.; MAFRA, Á. L.; GATIBONI, L. C. (ed.). Tópicos em ciência do solo. 7. ed. Viçosa: Sociedade Brasileira de Ciência do Solo, 2011. p. 119-170.

BARETTA, D. et al. Trap and soil monolith sampled edaphic spiders (Arachnida: Araneae) in Araucaria angustifolia forest. Scientia Agricola, Piracicaba, v. 64, n. 4, p. 375-383, 2007a.

BEEBY, A. Applying ecology. London: Chapman \& Hall, 1993. 441 p.

BERG, M. P.; HEMERIK, L. Secondary succession of terrestrial isopod, centipede, and millipede communities in grasslands under restoration. Biology and Fertility of Soils, Berlin, v. 40, n. 3, p. $163-170,2004$

BLAKE, G. R.; HARTGE, K. H. Bulk density. In: KLUTE, A. (ed.). Methods of soil analysis. Part 1. Physical and mineralogical methods. 2nd. ed. Madison: American Society of Agronomy and Soil Science Society of America, 1986. p. 363-375.

BROCARDO, C. R.; CÂNDIDO JÚNIOR, J. F. Persistência de mamíferos de médio e grande porte em fragmentos de floresta ombrófila mista no estado do Paraná, Brasil. Revista Árvore, Viçosa, v. 36, n. 2, p. 301-310, 2012.

BRUSSAARD, L.; RUITER, P. C.; BROWN, G. G. Soil biodiversity for agricultural sustainability. Agriculture, Ecosystems and Environment, Amsterdam, v. 121, n. 3, p. 233-244, 2007.

CASIDA JUNIOR, L. E.; KLEIN, D. A.; SANTORO, T. Soil dehydrogenase activity. Soil Science, Baltimore, v. 98, n. 6, p. 371-376, 1964.

CONG, W.-F. et al. Intercropping affects the rate of decomposition of soil organic matter and root litter. Plant and Soil, Berlin, v. 391, n. 1/2, p. 399-411, 2015.

COYLE, D. R. et al. Soil fauna responses to natural disturbances, invasive species, and global climate change: Current state of the science and a call to action. Soil Biology and Biochemistry, Oxford, v. 110, p. 116-133, 2017.

CROTTY, F. V. et al. Assessing the impact of agricultural forage crops on soil biodiversity and abundance. Soil Biology and Biochemistry, Oxford, v. 91, p. 119-126, 2015.

DECAËNS, T. et al. Factors influencing soil macrofaunal communities in post-pastoral successions of western France. Applied Soil Ecology, Amsterdam, v. 9, n. 1-3, p. 361-367, 1998. 
EMBRAPA. Manual de métodos de análise de solo. 2. ed. Rio de Janeiro: EMBRAPA Solos, 2011. $230 \mathrm{p}$.

EMBRAPA. Sistema brasileiro de classificação de solos. 2. ed. Rio de Janeiro: EMBRAPA Solos, 2006. 306 p.

GALLO, D. et al. Entomologia agrícola. Piracicaba: FEALQ, 2002. 920 p.

GEE, G. W.; OR, D. Particle-size analysis. In: DANE, J. H.; TOPP, G. C. (ed.). Methods of soil analysis. Part 4. Physical methods. Madison: Soil Science Society of America, 2002. p. 255-293.

GUERRA, M. P. et al. Exploração, manejo e conservação da araucária (Araucaria angustifolia). In: SIMÕES, L. L.; LINO, C. F. (ed.). Sustentável Mata Atlântica: a exploração de seus recursos florestais. São Paulo: SENAC, 2002. p. 85-102.

HUECK, K. As florestas da América do Sul. São Paulo: Polígono, 1972. 466 p.

HUTCHINS, M. et al.(ed.). Grzimek's animal life encyclopedia. 2nd. ed. Farmington Hills: Gale Group, 2003a. v. 2. 589 p.

HUTCHINS, M. et al. (ed.). Grzimek's animal life encyclopedia. 2nd. ed. Farmington Hills: Gale Group, 2003b. v. 3. 489 p.

JING, S. et al. Differences in Soil Arthropod Communities along a High Altitude Gradient at Shergyla Mountain, Tibet, China. Arctic, Antarctic, and Alpine Research, Boulder, v. 37, n. 2, p. 261-266, 2005.

LAVELLE, P.; SPAIN, A. V. Soil ecology. 1st. ed. Amsterdam: Kluwer Scientific, 2001. 678 p.

LIMA, A. C. R. et al. A functional evaluation of three indicator sets for assessing soil quality. Applied Soil Ecology, Amsterdam, v. 64, p. 194-200, 2013.

MAHARNING, A. R.; MILLS, A. A. S.; ADL, S. M. Soil community changes during secondary succession to naturalized grasslands. Applied Soil Ecology, Amsterdam, v. 41, n. 2, p. 137-147, 2009.

MARTIUS, C. et al. Microclimate in agroforestry systems in central Amazonia: does canopy closure matter to soil organisms? Agroforestry Systems, [s. l.], v. 60, n. 3, p. 291-304, 2004.

MAUNSELL, S. C. et al. Springtail (Collembola) assemblages along an elevational gradient in Australian subtropical rainforest. Australian Journal of Entomology, Canberra, v. 52, n. 2, p. 114-124, 2013.

MENTA, C. Soil fauna diversity - function, soil degradation, biological indices, soil restoration. In: LAMEED, G. A. (ed.). Biodiversity conservation and utilization in a diverse world. [S. l.]: INTECH, 2012. p. 59-94.

MOLINA, G. A. R.; POGGIO, S. L.; GHERSA, C. M. Epigeal arthropod communities in intensively farmed landscapes: Effects of land use mosaics, neighbourhood heterogeneity, and field position. Agriculture, Ecosystems and Environment, Amsterdam, v. 192, p. 135-143, 2014.

MOREIRA, M. et al. Arbuscular mycorrhizal fungal communities in native and in replanted Araucaria forest. Scientia Agricola, Piracicaba, v. 66, n. 5, p. 677-684, 2009.

MUMLADZE, L. et al. Oribatid mite communities along an elevational gradient in Sairme gorge (Caucasus). Experimental and Applied Acarology, Northwood, v. 66, n. 1, p. 41-51, 2015.

PEREIRA, J. M.; BARETTA, D.; CARDOSO, E. J. B. N. Fauna edáfica em floresta de Araucária. In: CARDOSO, E. J. B. N.; VASCONCELLOS, R. L. F. (ed.). Floresta com Araucária: composição florística e biota do solo. Piracicaba: FEALQ, 2015. p. 153-180.

PEREIRA, J. M. et al. Relationships between microbial activity and soil physical and chemical 
properties in native and reforested Araucaria angustifolia forests in the state of São Paulo, Brazil. Revista Brasileira de Ciência do Solo, Viçosa, v. 37, n. 3, p. 572-586, 2013.

PEREIRA, J. M. et al. Soil macrofauna as a soil quality indicator in native and replanted Araucariaangustifolia forests. Revista Brasileira de Ciência do Solo, Viçosa, v. 41, p. e0160261, 2017.

PONGE, J.-F. et al. The impact of agricultural practices on soil biota: a regional study. Soil Biology and Biochemistry, Oxford, v. 67, p. 271-284, 2013.

PONGE, J.-F. et al. Soil Macrofaunal communities are heterogeneous in Heathlands with different grazing intensity. Pedosphere, [s. l.], v. 25, n. 4, p. 524-533, 2015.

RIBEIRO, M. C. et al. The Brazilian Atlantic Forest: How much is left, and how is the remaining forest distributed? Implications for conservation. Biological Conservation, Cambridge, v. 142, n. 6, p. 1141-1153, 2009.

RÖDER, J. et al. Heterogeneous patterns of abundance of epigeic arthropod taxa along a major elevation gradient. Biotropica, [s. l.], v. 49, n. 2, p. 217-228, 2017.

ROUSSEAU, L. et al. Soil macrofauna as indicators of soil quality and land use impacts in smallholder agroecosystems of western Nicaragua. Ecological Indicators, [s. l.], v. 27, p. 71-82, 2013.

ROŹEN, A. et al. Soil faunal activity as measured by the bait-lamina test in monocultures of 14 tree species in the Siemianice common-garden experiment, Poland. Applied Soil Ecology, Amsterdam, v. 45, p. 160-167, 2010.

SANTOS, R. L. R. et al. Comunidade arbórea de trecho de floresta secundária com araucária na Estação Ecológica de Bananal, Bananal-SP. Instituto Florestal, São Paulo, v. 40, p. 137-142, 2009.

SANTOS, R. L. R.; IVANAUSKAS, N. M. Estrutura do componente arbóreo de trecho de floresta de araucária na Estação Ecológica de Itaberá, Itaberá-SP, Brasil. Instituto Florestal, São Paulo, v. 42, p. 127-131, 2010.

SAS INSTITUTE. SAS/STAT: user's guide statistics. [S. l.]: CarySAS Institute, 2002.

SOUZA, R. P. M. Estrutura da comunidade arbórea de trechos de florestas de Araucária no estado de São Paulo, Brasil. 2008. Dissertação (Mestrado em Recursos Florestais) - Universidade de São Paulo, Piracicaba, 2008.

TER BRAAK, C. J. F.; ŠMILAUER, P. CANOCO reference manual and CanoDraw for Windows User's Guide: software for canonical community ordination (version 4.5). Wageningen: Microcomputer Power, 2002. 10 p.

VAN RAIJ, B. et al. Análise química para avaliação da fertilidade de solos tropicais. Campinas: Instituto Agronômico de Campinas, 2001. 285 p.

VANCE, E. D.; BROOKES, P. C.; JENKINSON, D. S. An extraction method for measuring soil microbial biomass C. Soil Biology and Biochemistry, Oxford, v. 19, n. 6, p. 703-707, 1987.

VASCONCELLOS, R. L. F. et al. Soil macrofauna as an indicator of soil quality in an undisturbed riparian forest and recovering sites of different ages. European Journal of Soil Biology, Amsterdam, v. 58, p. 105-112, 2013.

ZEPPELINI, D. et al. Collembola as bioindicators of restoration in mined sand dunes of Northeastern Brazil. Biodiversity and Conservation, New York, v. 18, n. 5, p. 1161-1170, 2009. 